\title{
EL INCENDIO DEL TEATRO DE ZARAGOZA EN 1778
}

Roger ALIER

Universidad de Barcelona

Uno de los peores enemigos de la ópera y de los teatros han sido los incendios. Casi todos los grandes teatros de ópera europeos han tenido que pagar un alto tributo al fuego, a veces con un doloroso coste añadido en vidas humanas. El Teatro de la Santa Cruz (1787), el Covent Garden de Londres (1856), el Teatro Petruzzelli de Bari (1991), que fue objeto de un incendio intencionado, el Gran Teatre del Liceu (1861 y 1994) y, más recientemente aún, el Teatro de la Fenice de Venecia (1996), son algunos de los locales más famosos que han iluminado con sus llamaradas siniestras una noche de su historia.

Hemos traído a estas páginas una curiosa descripción de un incendio hoy virtualmente olvidado: el que afectó al Coliseo de Zaragoza, en el año 1778, en tiempos de Carlos III. El relato, realizado por el cronista oficial de la capital aragonesa, Tomás Sebastián y Latre, tiene la vivacidad y la garra del suceso vivido por quien lo narra y da detalles sumamente curiosos que son espejo fiel de unas costumbres teatrales y sociales que no dudamos serán de interés para nuestros lectores. En este artículo reproducimos la parte más interesante de este antiguo texto y hemos conservado la ortografía original, pues su lectura no entraña dificultades y contribuye a conservarle el sabor de la época.

El Teatro de Zaragoza dependía del Hospital de la Ciudad, como sucedía en la mayor parte de las poblaciones españolas, pues en esta época en que la Iglesia todavía ejercía una fuerte influencia sobre las costumbres, la existencia de los teatros se tenía que "justificar" a base de que sus ingresos tuviesen una finalidad caritativa o asistencial. El nuevo teatro de la Real Sitiada (la entidad gestora del Hospital) había reemplazado la antigua Casa de las Comedias de Zaragoza, que había tenido muchos años de existencia. Ya en ésta la ópera había sido un género habitual en la ciudad desde hacía por lo menos veinte años; el nuevo local había acogido los espectáculos italianos y era, en la descripción del propio cronista:

"reputado por uno de los mejores de España, por la situacion, comodidad de entradas y salidas, capacidad para 1300 personas, y buena distribucion como demuestra su Planta, à que se juntaba su adorno interior de columnas y pintura que lo hacian sumamente vistoso. Esta fabrica se 
construyò en el mismo sitio en que estaba la antigua Casa de Comedias, que habia levantado el Santo Hospital General el año 1589, con permiso que pidiò para ello à la Ilustrissima Ciudad; habiendose aplicado desde entonces à beneficio de los pobres enfermos todo su producto como de fundo propio. La Real Sitiada comisionò para la direccion de la obra à su Regidor el Excelentissimo Señor Conde de Sàstago, à cuyo zelo y actividad se debiò, que empezandose à trabajar el dia 26 de Mayo de 1769, se representase ya en el nuevo Teatro à 7 de octubre del mismo año."

Desde hacía una veintena de años, la ópera italiana se había convertido en un género muy apreciado del público zaragozano, que llenaba las sesiones de ópera con especial interés. Como en otras ciudades españolas, en la década de los años 1760 se había aclimatado este género con representaciones de óperas serias de corte todavía tardo-barroco, pero estaban adquiriendo cada vez mayor sufragio del público las óperas bufas de las escuelas napolitana y veneciana. Sin embargo, igual que en otras ciudades de España, esta afición no se introdujo sin oposición de los sectores más reaccionarios, que escribieron con sus dicterios un capítulo nada despreciable de la historia de las controversias sobre la licitud del teatro que historiaría en su momento Emilio Cotarelo y Mori ${ }^{1}$. En efecto, en 1766, un religioso, el P. Nicolás Blanco, escribió un libro furibundo: Examen Theológico moral sobre los Theatros actuales de España ${ }^{2}$, en el que ponía como ejemplo de los peligros morales que entrañaban los espectáculos operísticos la ópera de Galuppi Il filosofo di campagna, representada poco antes en Zaragoza, puesto que en ella aparecía en escena una muchacha que desobedeciendo las órdenes de su padre, y utilizando enredos y medias verdades, se las ingeniaba para rechazar el candidato matrimonial impuesto por éste y acababa casándose con el joven de su elección. Faltaban cuarenta años para que Moratín dijera todo lo contrario en El sí de las niñas.

A pesar de la gravedad de las acusaciones del P. Blanco, no parece que se hiciera cambio alguno en las costumbres teatrales zaragozanas, y en el decenio siguiente continuaron las compañías italianas ofreciendo óperas de todos los tipos, combinadas con otros espectáculos teatrales, en el Coliseo de Zaragoza. El público rubricaba con su asistencia masiva la opinión favorable que tenía de los espectáculos operísticos.

Pero en 1778 el nuevo Teatro se encontró con su destino en la forma del aparatoso incendio que el cronista nos describe en las páginas siguientes, y que supuso una verdadera catástrofe que alcanzó a todas las clases sociales, pues como consecuencia de las quemaduras falleció incluso el propio capitán general de Aragón, D. Antonio Manso.

1. Cotarelo y Mori, Emilio: Bibliografía de las controversias sobre la licitud del teatro en España. Madrid, Tip. de la "Revista de Archivos, Bibliotecas y Museos", 1934.

2. Blanco, Nicolás: Examen Theolgico-moral sobre los Theatros actuales de España. Zaragoza, Imp. de Francisco Moreno, 1766. 


\section{RELACION HISTÓRICA DE LOS SUCESOS OCURRIDOS EN ZARAGOZA CON MOTIVO DEL INCENDIO DE SU COLISEO EN LA NOCHE DEL DOCE DE NOVIEMBRE DE 1778.}

ESCRITA SOBRE DOCUMENTOS AUTÉNTICOS Y NOTICIAS FIDEDIGNAS, DE ORDEN DE LA ILLM ${ }^{\text {a }}$ CIUDAD POR SU CORONISTA D. THOMAS SEBASTIAN Y LATRE, del Consejo de su Magestad su Secretario, y Socio de la Real Sociedad Aragonesa de Amigos del País.

Quien la dedica a su $\mathrm{Illm}^{\circ}$ Ayuntamiento.

ZARAGOZA, AÑO 1779. En la imprenta de Francisco Moreno. CON LAS LICENCIAS NECESARIAS

En la tarde del dia 12. de Noviembre del año proximo pasado, añadia gravedad y lucimiento al magnifico Coliseo de esta Ciudad un número muy considerable de personas de ambos sexos, en especial de las primeras clases, asi por ser el dia de gala, como porque la Compañia de Operistas Italianos tenia anunciada una pieza cèlebre con iluminacion è intermedios de bello gusto; cuya execucion habia grangeado otras veces à los Actores particulares satisfacciones del Pùblico.

Representabase La Real Jura de Artaxerxes, y concluido el segundo Acto cerca de las seis y quarto, se preparaba acceleradamente una decoracion de Jardin, que habia de servir para el Bayle de Las Estatuas animadas, quando por imprudencia ò por descuydo de los Mozos que manejaban los bastidòres, se inclinó alguna vela àcia una fuente que estaba en medio del Jardin, y prendiò en ella fuego con la rapidèz que era consiguiente à la gran disposicion de las materias de que constaban los cuerpos.

Un accidente de esta naturaleza puso à todos los Actores y asistentes en aquel cuydado y movimiento que era regular. Pero como estaba echado el primer telon, segun costumbre, ninguno de los Espectadores tuvo noticia del suceso; pues aunque se percibia algun estrepito y griterìa,se creyò todo efecto de los altercados que ordinariamente ocurren entre los que disponen las mutaciones; hasta que saliendo precipitadamente por el lado izquierdo del tablado una Baylarina gritò, foco: aviso oportuno, si no se hubiera malogrado por la turbacion y poca voz de quien la daba, en un momento en que no reynaba el silencio que se requeria. Asi no executò sino la curiosidad de los menos distantes, por saber con certeza lo que habia prevenido.

Luego se saliò de la duda presentandose el primer Actor, y diciendo en voz esforzada y clara: foco, foco. Pero apenas se habia retirado dexando la Casa llena de susto y commocion, quando para calmarla salio el Impresario diciendo: Señores no es nada, ya està apagado el fuego. Y esto se creyò tan cierto por algunos que bolvieron à ocupar sus puestos. El que lo asegurò estaba persuadido de que era verdad; y lo era en quanto à la fuente que con la mayor prontitud derribaron y deshicieron: mas la turbacion, junta al deseo de evitar toda mala consequencia, no le dexaron advertir que el ayre habia elevado las llamas à las bambalinas y telar en que estaban colgados los bastidòres. Consiguientemente ardia ya la parte superior del foro, y comunicandose de 
alli el fuego à lo restante (lo que se veìa con bastante claridad sin embargo de estar aun caìdo el telon) empezaron las gentes à ponerse en fuga, suspendiendola solo los que tuvieron por acertado caminar lentamente, por libertarse del tropel inescusable que en semejantes lances suele estorvar los remedios y aumentar las desgracias.

En pocos instantes, y sin grave daño se despoblaron Lunetas, Gradas y Patio à beneficio de las muchas salidas que tenian proximas à la calle. No las hallaron tan prontas y faciles las personas que habia en los Palcos, asi por la mayor distancia, como porque el cariño, el respeto y otras obligaciones aumentaban las dificultades de salvarse. Pero aun llegaron estas à ser mayores para las Mugeres que se hallaban en el Gallinero ò Cazuela, que era el sitio mas alto de la Casa; pues las que, ò no percibieron ò por menos temerosas no aprovecharon los primeros avisos, se detuvieron mas de lo que convenia à su delicadeza y situacion.

Crecìa por instantes la confusion y el riesgo. Mutuamente se atropellaban y se impedian la fuga las gentes que baxaban de los Aposentos; y los Actores, que habiendo podido escaparse del bolcan en que se abrasaba el Vestuario, corrian por los transitos buscando la escalera principal. Todo era suspiros, ayes y gritos, pidiendo socorro à los hombres, y à Dios misericordia. Todo era susto y horror.

Llegò este à lo sumo, quando las arañas de cristal, cortadas las cuerdas por las llamas, cayeron con un estrepito y crugido espantoso; y quando despues de encendido el primer telon, el ayre que por la puerta de los Comicos entraba en el Teatro, no encontrando desahogo por los despedideros le buscò por los Palcos y ventanas proximas à la escalera, apagando las luces y llevando consigo globos de fuego y densas nubes de humo fétido.

Entonces la lobreguèz acabò de desmayar à unos, y el ayre pestilencial que se respiraba privò de sentido à otros; de suerte que fueron muy pocos los que ò por hallarse casualmente en sitio algo defendido, ò por una firmeza extraordinaria, se mantuvieron con espiritu; y de ellos cada uno creìa ser el unico que habia quedado vivo: asi lo persuadiò el no tocar sino cuerpos sufocados y el profundo silencio que se advertia por todas partes, sin percibirse ni un suspiro.

En los pocos minutos que sucedian estas cosas, el inopinado y ràpido progreso que hacia el fuego comunicandose al Hospital por su immediacion, y amenazando las casas vecinas, dificultaba el atender à un tiempo à todas partes. Pero como el Señor D. Diego Navarro y Gomez, Cavallero Corregidor de esta Ciudad, que presidia en el Teatro, saliò à las primeras voces (aunque con bastante trabajo porque ya las gentes se lo embarazaban) tomò desde luego, con la actividad y prudencia que permitian las circunstancias, las disposiciones convenientes para socorrer las necesidades graduandolas segun la urgencia. Y hallandose alli los Escribanos del Juzgado Ordinario y sus Alguaciles, en un instante se distribuyeron las ordenes para tocar à fuego en la Parroquia mas immediata, que es la de San Gil, y el relox de la Ciudad, que es el de la Torre nueva: (2) se echò pregon para que acudiesen baxo graves penas Albañiles, Carpinteros y Aguadores: (3) se providenciò llamar Medicos y Cirujanos: se hizo traer hachas de viento: y finalmente se mandò, que todo genero de oficiales y trabajadores acudieran con azadas y espuertas, para estar prontos à quanto pudiera ocurrir. 
Muy à los principios de estas providencias llegaron los Señores Alcaldes del Crimen, y conformandose con las tomadas hasta entonces, añadieron otras segun lo pedian los lances, y con infatigable constancia no perdonaron trabajo alguno para atender à los tristes objetos que se les presentaban; contribuyendo à ello el Señor Alguacil Mayor de la Real Audiencia, el Alcalde Mayor de esta Ciudad, D. Luis Gorron de Contreras, y el Estado mayor de la Plaza que procurò dar por su parte todos los auxilios correspondientes.

Como en las aflicciones pùblicas nunca faltan personas que señaladamente den pruebas de su espiritu, las hubo tambien en esta ocasion, pues fueron muchos los que de varios modos contribuyeron al socorro de este general conflicto. D. Joaquin Texero, Alferez agregado à esta plaza, y Diego Polo subieron por una escalera de mano à uno de los balcones de los que caìan à los corredores por donde se iba à la Cazuela; y encontrando algunas Mugeres que pedian socorro (à quienes daba fuerzas la necesidad) las echaron por el balcon à la calle, en la que esperaban ya otros que las recibian para que no cayeran al suelo; y aunque intentaron pasar adelante, el fuego y el humo que se extendia por todas partes los precisò à bajar por donde habian subido; y las mismas dificultades encontraban en la puerta. Sin duda por buscarla y huìr del riesgo se atropellaban las Mugeres y se cerraban el paso, lo que fue causa de que pereciera un numero bastante considerable.

Mientras continuaban estos en procurar los socorros que les presentaba la ocasion, acreditando en ellos el Oficial Texero su esfuerzo, teson y caridad; Joseph Frayle, y Joseph Gavàs, Maestros Carpinteros, aventurandose al riesgo por libertar à tantos Conciudadanos como veian en la ultima afliccion, tomaron hachas de viento, y con esta luz empezaron à desembarazar la entrada de la puerta de Aposentos, en la que habia tanta gente amontonada que formando una gran balla hacian impenetrable aquel paso; el que era forzoso desahogar para sacar los que ocupaban la escalera, y subir despues à los corredores. Sacaban à unos muertos, à otros heridos, y à los mas medio sufocados; pero no faltaban personas zelosas que luego cuydasen de ellos. Despejado aquel corto recinto, cuya vista pudiera angustiar al más animoso corazon, advirtieron la imposibilidad de subir por la escalera de la Casa, sin causar grave daño à las muchas gentes que estaban tendidas en ella; y aun sin el de algunas, que habiendose sostenido vigorosamente hasta entonces no les dexaban baxar, executando su ternura y compasion, y alternando con tristes y fervorosas deprecaciones en llamar à los Santos, en ayudarse à bien morir, y generalmente en implorar el amparo de nuestra Patrona Maria Santissima del Pilar. Para vencer pues estas dificultades traxeron escaleras de mano, las arrimaron à la barandilla de la principal, con lo que subian por encima de ella y baxaban por las otras. Huvo algunas Señoras y muchos Cavalleros que no pudiendo llegarles este socorro se determinaron à arrojarse por la caxa de la escalera, fiando en la ayuda de los que entraban à contribuir à esta grande obra; entre los que se distinguieron por su bizarria y animosidad algunos Oficiales Militares que de su resulta han estado expuestos à perder la vida.

Con los que salian por este medio y los muertos ò casi sufocados que sacaban de la escalera (4), se iba abriendo el camino para los corredores; y tres gradas antes de llegar à ellos encontraron al Excelentissimo Señor Capitan General de este Reyno y Presidente de su Real Audiencia, cuyo notorio espiritu y zelo por el servicio del Rey y del Pùblico le detuvieron 
demasiado en el Falco, persuadido de que su respeto y autoridad contribuirian con sus providencias à atajar el fuego, (5), ò por lo menos à evitar el tropèl que creyò indispensable si veìan que su Exc. dexaba su puesto: por cuya razon lo sacaron muy maltratado del fuego y del humo; de modo que ya fue forzoso llevarlo à casa de la Excelentissima Señora D. Cathalina de Huarte y Silba, porque el Palacio estaba mas distante, y era preciso atender luego à reparar el grande daño que se le advertia.

El incendio habia tomado ya tal cuerpo que casi lo ocupaba todo, y conforme iba estrechando el terreno acortaba tambien el tiempo para las esperanzas y los socorros; tanto que para salvar algunos pocos no se hallò ya otro remedio que el de arrojarlos por la escalera rodando, por si habia abajo quien los sacase al Coso. Muchas fueron las personas que desde el principio se dedicaron à tan pesada ocupacion, conduciendo los estropeados y los muertos à las casas de enfrente, ò dexandolos encomendados en la calle à sujetos que inflamados del mismo zelo, se los arrebataban de las manos. En todas estas faénas no dexaba de animar bastante la presencia y la voz de algunos personages; entre ellos muy particularmente por su alta calidad los Señores Conde de Sàstago y D. Ramon Pignateli quienes casi desde los primeros instantes asistieron à las puertas del Teatro, donde à su Exc. por Regidor del Hospital se le doblaban los cuydados y las penas, viendo reducirse à cenizas uno de los mas utiles fundos de los Pobres, ya que á estos se les iba acercando el fuego por aquella parte de la fabrica mas contigua à sus Quadras.

El Regimiento de Caballeria de Calatraba estaba desmontado, y con los Fusileros del Reyno formaban un cordon que ocupaba un largo trecho del Coso, teniendo à mas tomadas todas las bocas de las calles, y apostadas centinelas en los sitios necesarios para contener las avenidas de las gentes, facilitar el paso de las que podian ser utiles de qualquier modo, y evitar el tropel de unos y de otros.

En aquel dilatado campo bullìa un enxambre numeroso de Ciudadanos de diferentes clases y estados, procurando socorros à sanos y enfermos. Algunos Señores Oydores y los Caballeros Regidores de la Ciudad que se hallaban en disposicion de asistir, discurrian por todas partes contribuyendo con sus luces y facultades (6) al perfecto cumplimiento de las providencias que se iban tomando, y executaban con prontitud los Alcaldes de Barrio, los Alguaciles y Porteros de la Real Audiencia y Juzgado, entre los quales se distinguieron algunos por su desembarazo y tesòn. Los Alarifes y Carpinteros se empleaban en atajar el fuego, cortando por donde amenazaba mayor peligro. Los Aguadores acudian à apagarlo, y al mismo tiempo se iban desocupando las casas vecinas, cuyos muebles se trasladaban à las mas seguras, ò se amontonaban en la calle dexandolos à la custodia de la Justicia, de la Tropa, y de varias personas de confianza que se dedicaron à recoger las ropas y alhajas de los desgraciados. Los Medicos y Cirujanos aprovechaban los instantes, para aplicar los remedios à tantos como los necesitaban à un tiempo.

Las llamas subian tan altas que segun se supo despues, se veian à quatro leguas y mas de distancia. Asi no serà exageracion decir que llegaron à iluminar toda la Ciudad, extendiendo los recelos hasta los parages mas distantes las chispas y materias encendidas que llevaba el ayre; y esto junto al toque incesante de Campanas atrahia al Coso un gran numero de pueblo de todas 
clases que pocas veces ha visto mayor. El ansia de socorrer cada uno à las personas que le interesaban, era causa de que quantas calles guian à la del Coso estuvieran inundadas no solo de los que venian à ella à buscar su desengaño, sino de los que ya se retiraban llorando su desgracia; cuyos tristes suspiros y penetrantes ayes, eran fatales anuncios que hacian recelar funestas consequencias à los fieles Esposos, à los tiernos Padres, à los buenos Hijos, y à los verdaderos Amigos, que animados del cariño del amor, del respeto y de amistad, despreciaban los peligros por encontrar à quien los empeñaba en ellos.

\section{Notas}

2.- Esta campana se oye de toda la Ciudad y gran parte de su Huerta: pesa 260 quintales y està puesta en una Torre aislada de 416 palmos de elebacion y 60 de diametro...

3.- Este Gremio es de los que deben acudir mas pronto à los incendios, por no haber en el casco de la Ciudad fuentes ni depositos pùblicos de agua. Cada uno debe tener un naype ò carton con su nombre y apellido, y el que acude primero presentandolo al Ministro superior, ò Alcalde de Barrio que se halla en la quema, gana un premio en dinero, à arbitrio de la Justicia y à costa del que llega el ultimo...

4.- Me vi en ella entre una multitud de desgraciados que aumentaban la angustia y la afliccion. Todo el Pueblo fue testigo de lo mucho que trabajaron entonces los dos honrados vecinos Fraile, y Gavàs, y yo les debì la caridad de que ayudados de un fiel Criado mio que no me desamparò exponiendo muchas veces su vida, me sacasen del riesgo en que estuve, superior al de todos los concurrentes por mi constitucion fisica: y me conduxesen al Café de Carmen Montanino, que por estar frente al Teatro y tener grandes proporciones para el mas pronto socorro sirviò de general asilo.

5.- En todo el tiempo de su gobierno acreditò su Exc. constantemente esta verdad. (...) Por el grande amor que tenia à sus semejantes, como por estar justamente persuadido de que una de las especiales obligaciones de su alto ministerio era acudir prontamente à qualquiera calamidad pùblica, corrìa el primero à los incendios, aunque fuera en las horas mas incomodas de la noche, y en llegando al lugar de la desgracia tomaba siempre acertadissimas providencias, que descubrian su presencia de animo, capacidad y experiencia, sin retirarse jamas hasta verlos cortados con entera seguridad. Y en el fin no pudo dar prueba mas sobresaliente que la de mantenerse el ultimo en su Palco para tomar de mas cerca las disposiciones convenientes, y evitar con su voz y su presencia las tropelias y estragos que conocia iban à seguirse: como lo refiere con admiracion y sentimiento el Coronel agregado à esta Plaza D. Dionisio Soler, quien no se apartò del lado de su Exc. desde el principio del fuego hasta que en el conflicto de la escalera vinieron à perderse cercados de humo y llamas, de que este animoso Oficial tuvo la fortuna de salvarse, aunque maltratado. 
6.- El Señor Intendente de este Reyno ayudò tambien con las suyas quanto le fue posible desde el punto que saliò del Teatro, para lo que se vio en bastante afliccion.

Esta "Relación histórica" continúa con la descripción de las precauciones que hubo que tomar para que el fuego no se propagase al vecino Hospital, el traslado de los enfermos a otros locales y a un convento, la colocación de los cadáveres en la calle, la labor que hubo que realizar para atajar un nuevo rebrote del incendio que ocurrió en la madrugada, y que amenazó de nuevo al Hospital, y la suspensión de la ejecución de dos reos prevista para la madrugada siguiente, para evitar más muertes en la ciudad. A los reos les fue conmutada la pena por diez años de presidio en Africa. El capitán general Antonio Manso falleció el día 15 por la mañana como consecuencia de las graves quemaduras recibidas. El opúsculo comenta luego los rumores que corrieron por la ciudad sobre el numero elevadísimo de muertes acaecidas; en parte el opúsculo se editó por el deseo de las autoridades de dar su versión sobre el verdadero alcance del incendio, que según el cronista se cobró en total 77 muertos y dejó lesiones notables en 52 personas. Son cifras un tanto moderadas para el cuadro dantesco que se describe y es muy posible que fueran más y no se dijera por la actitud oficial de disimular la tragedia - que es algo que se percibe también en otras partes del relato, como la prohibición de que las iglesias tocaran a muertos. Las consecuencias del incendio fueron la decisión de suspender para siempre los espectáculos teatrales, pues los enemigos de las mismas estaban siempre al acecho de la menor ocasión para conseguir su erradicación, como habían logrado en Sevilla y, durante un tiempo prolongado, en Valencia. Sin embargo, pocos años después encontramos nuevamente en activo la vida teatral de Zaragoza, incluyendo las representaciones de ópera, tan denostadas por algunos. 